\title{
BMC
}

Evolutionary Biology

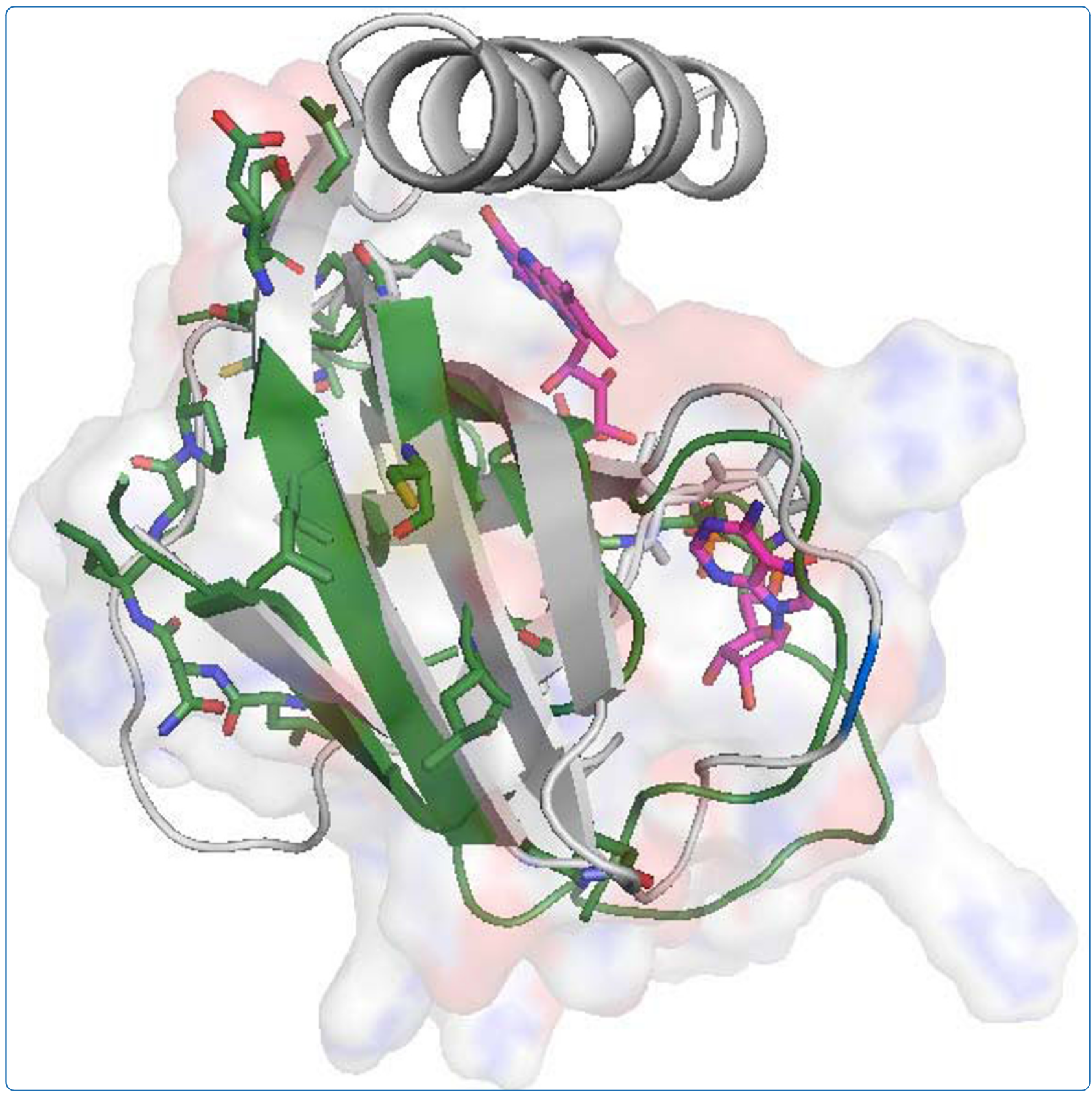

\section{Evolutionary divergence of chloroplast FAD synthetase proteins}

Yruela et al. 


\title{
Evolutionary divergence of chloroplast FAD synthetase proteins
}

Inmaculada Yruela ${ }^{1,2^{*}}$, Sonia Arilla-Luna ${ }^{2,3}$, Milagros Medina ${ }^{2,3}$, Bruno Contreras-Moreira ${ }^{1,2,4}$

\begin{abstract}
Background: Flavin adenine dinucleotide synthetases (FADSs) - a group of bifunctional enzymes that carry out the dual functions of riboflavin phosphorylation to produce flavin mononucleotide (FMN) and its subsequent adenylation to generate FAD in most prokaryotes - were studied in plants in terms of sequence, structure and evolutionary history.

Results: Using a variety of bioinformatics methods we have found that FADS enzymes localized to the chloroplasts, which we term as plant-like FADS proteins, are distributed across a variety of green plant lineages and constitute a divergent protein family clearly of cyanobacterial origin. The C-terminal module of these enzymes does not contain the typical riboflavin kinase active site sequence, while the $\mathrm{N}$-terminal module is broadly conserved. These results agree with a previous work reported by Sandoval et al. in 2008. Furthermore, our observations and preliminary experimental results indicate that the C-terminus of plant-like FADS proteins may contain a catalytic activity, but different to that of their prokaryotic counterparts. In fact, homology models predict that plant-specific conserved residues constitute a distinct active site in the C-terminus.

Conclusions: A structure-based sequence alignment and an in-depth evolutionary survey of FADS proteins, thought to be crucial in plant metabolism, are reported, which will be essential for the correct annotation of plant genomes and further structural and functional studies. This work is a contribution to our understanding of the evolutionary history of plant-like FADS enzymes, which constitute a new family of FADS proteins whose C-terminal module might be involved in a distinct catalytic activity.
\end{abstract}

\section{Background}

Flavin mononucleotide (FMN) and flavin adenine dinucleotide (FAD) are essential cofactors for numerous enzymes (i.e., dehydrogenases, oxidases, reductases) that participate in one- and two-electron oxidation-reduction processes critical to the major metabolic routes in all living organisms [1-4]. Riboflavin (RF), the precursor of FMN and FAD can be de novo synthesized by plants, fungi and many bacteria, but in mammals the only known RF source is the exogenous riboflavin (vitamin $\mathrm{B}_{2}$ ) obtained through the diet [5-7].

In most prokaryotes, the synthesis of FMN and FAD is catalyzed from RF and ATP by a single bifunctional enzyme, usually known as FAD-synthetase (FADS), through the sequential action of its two enzymatic

\footnotetext{
* Correspondence: yruela@eead.csic.es

'Estación Experimental de Aula Dei, Consejo Superior de Investigaciones Científicas (CSIC), Avda. Montañana, 1005, 50059 Zaragoza, Spain Full list of author information is available at the end of the article
}

activities: an ATP:riboflavin kinase (RFK, EC 2.7.1.26) that transforms RF and ATP into FMN, and an ATP: FMN adenylyltransferase (FMNAT, 2.7.7.2) that catalyzes the subsequent adenylylation of FMN to FAD. Thus, FADS is a bifunctional RFK/FMNAT enzyme [8]. FADSs are typically $310-340$ residues in length and are folded in two modules [9-11], each one mainly involved in one of the activities. The RFK reaction has been related with the $C$-terminal module (RFK-module), while the $\mathrm{N}$-terminal module is mainly related to the FMNAT activity (FMNAT-module); hence, two independent substrate binding and catalytic sites are in charge of each activity $[11,12]$. In one hand, the RFKmodule $(\sim 180$ aa) folds in a globular domain and its overall topology is similar to that found in the RFKs from Homo sapiens (HsRFK) and Schizosaccharomyzes pombe ( $S p$ RFK), with differences only observed in the loops connecting secondary structure elements $[13,14]$. Furthermore, the substrate binding motifs PTAN and 
GxY of the RFK-module are conserved among FADSs and eukaryotic RFKs. In the other hand, the FMNATmodule consists of an $\alpha / \beta$ dinucleotide binding domain with a typical Rossmann fold topology ( 150 aa) [9-11]. Moreover, it seems to be remotely similar to the nucleotidyltransferase (NT) superfamily and contains the typical $(\mathrm{H} / \mathrm{T}) \mathrm{xGH}$ and $\mathrm{xSST} / \mathrm{SxxR}$ motifs involved in binding nucleotide and phosphate groups. Interestingly, monofunctional enzymes with only RFK activity have been described in Bacillus subtilis [15] and Streptococcus agalactiae [16] but no monofunctional FMNAT enzymes have been reported in prokaryotes.

A different scenario is found in eukaryotes, where both activities are generally split in two different enzymes with either RFK or FMNAT activity [17-20]. As mentioned above, the RFK enzymes show sequence and structure similarity to the RFK-module of prokaryotic FADS [13,14]. However, eukaryotic FMNATs share little or no sequence similarity to the FMNAT-module of FADS, as these enzymes belong to two different protein superfamilies, which are thought to require different sets of active-site residues to carry out the same chemistry [21-23]. The eukaryotic FMNAT-module is currently classified as a member of the 3'-phosphoadenosine 5'phosphosulfate (PAPS) reductase-like family belonging to the "adenosine nucleotide $\alpha$-hydrolase-like" superfamily, which has motifs different from those of NTs.

In plants only a few efforts have been devoted to this system. Early studies characterized apparently monofunctional enzymes with either RFK or FMNAT activities in several plant species [24-27]. In those studies the subcellular localization of RFK and FADS was not addressed although it is known that plants use flavin nucleotides in mitochondria, plastids and in the cytosol. In an earlier work RFK activity was associated to the cytosol and to an organellar fraction containing chloroplasts and mitochondria [28]. More recently, a bifunctional enzyme with both FMN hydrolase and RFK activities has been described in Arabidopsis thaliana (AtFHy/RFK) [29], whose $\mathrm{N}$-terminal module responsible for the FMN hydrolase activity, shares sequence similarity with members of the haloacid dehalogenase (HAD) superfamily. AtFHy/RFK enzyme was predicted to be cytosolic [29]. Additionally, two more enzymes with FMNAT activity have been identified, cloned and characterized in the same species [30]. These AtRibF1 and $A t$ RibF2 enzymes, herein plant-like FADS proteins, have an $\mathrm{N}$-terminal module which is found to be homologous to the FMNAT-module of FADS, but instead its $C$-terminal module does not catalyze RF phosphorylation. AtRibF1 and AtRibF2 were localized to the chloroplast [30]. In mitochondria, the catalytically conversion of RF into FMN and FAD has been reported, due to the existence of mitochondrial RFK and FADS enzymes
[31], but nevertheless FADS activity was much lower than in chloroplasts. These results agree with the cited confocal microscopy studies [30], but the hypothesis for the localization of FADS isoforms (AtRibF1 and AtRibF2) in mitochondria cannot be ruled out on the basis of bioinformatics (TAIR) analysis [31]. The mitochondrial FAD-forming enzymes reside in two distinct monofunctional enzymes, which can be separated in soluble and membrane-enriched fractions. It is worth mentioning that the genes encoding organellar RFK activity remains unidentified.

In order to investigate RFK and FMNAT activities in plants we have conducted an extensive bioinformatics survey using the available genomes in public databases. Here we report the identification of a conserved $C$-terminal module in plant FADS enzymes, which does not contain the typical RFK active site sequence, suggesting that it belongs to a new family of FADS proteins. The activity of this module is discussed.

\section{Results and Discussion}

\section{Sequence and evolutionary analysis}

As shown in Table 1, most prokaryotic genomes (1178/ 1194) surveyed in this study, including cyanobacteria, contain a single gene encoding for a bifunctional FADS enzyme, hereafter identified as FADS-type I protein. Sequence searches in a variety of repositories of green plant sequences allowed us to identify a related group of

Table 1 Bacterial genomes containing FADS-like proteins ${ }^{(1)}$

\begin{tabular}{|c|c|c|}
\hline Type of protein & Genomes & $\mathbf{N}$ \\
\hline $\begin{array}{l}\text { FMNAT } \\
\text { RFK }\end{array}$ & $\begin{array}{l}\text { Chthoniobacter flavus Ellin428 ctg76 } \\
\text { Mesoplasma florum L1 } \\
\text { Mycoplasma capricolum subsp. capricolum } \\
\text { Mycoplasma mycoides subsp. capricolum }\end{array}$ & 4 \\
\hline FADS-type I & Most bacterial genomes & 1171 \\
\hline $\begin{array}{l}\text { FADS-type I } \\
\text { FMNAT }\end{array}$ & $\begin{array}{l}\text { Bacillus cereus 03BB102, } \\
\text { Bacillus cereus ATCC } 10987 \\
\text { Bacillus turingiensis str. AlHakam } \\
\text { Geobacillus thermodenitrificans NG80-2 } \\
\text { Listeria monocytogenes EGD-e }\end{array}$ & 5 \\
\hline $\begin{array}{l}\text { FADS-type I } \\
\text { RFK }\end{array}$ & $\begin{array}{l}\text { Bacillus subtilis subsp. subtilis } \\
\text { Haemophilus influenzae } 86-028 \mathrm{NP}\end{array}$ & 2 \\
\hline $\begin{array}{l}\text { FADS-type I } \\
\text { FADS-type II }\end{array}$ & $\begin{array}{l}\text { Arthrobacter chlorophenolicus A6 } \\
\text { Bacillus cereus subsp. cytotoxis NVH 391-98 } \\
\text { Lactobacillus plantarum JDM1 } \\
\text { Lactobacillus plantarum WCFS1 } \\
\text { Listeria monocytogenes HCC23 } \\
\text { Listeria welshimeri serovar } \\
\text { Oceanobacillus iheyensis HTE831 } \\
\text { Treponema denticola ATCC } 35405\end{array}$ & 8 \\
\hline FADS-type II & $\begin{array}{l}\text { Eubacterium saphenum ATCC } 49989 \\
\text { Mycoplasma conjunctivae } \\
\text { Treponema pallidum subsp. pallidum str. Nichols }\end{array}$ & 3 \\
\hline $\begin{array}{l}\text { FADS-type } \| \\
\text { RFK }\end{array}$ & Alistipes putredinis DSM 17216 & 1 \\
\hline
\end{tabular}

${ }^{1}$ Check details in section "Sequence analysis" of Methods. 
genes, which contain two domains with high similarity (see Methods section) to FADS-type I sequences (Figure 1 and 2), and are also present in a single copy in most cases with currently available complete genome (14/18). This result agrees with previous work by Sandoval et al. [30]. The $\mathrm{N}$-terminal module of these proteins displays high similarity to the FMNAT-module of prokaryotic FADStype I, showing the typical motifs $\mathrm{HxGH}$ and $\mathrm{xSST} / \mathrm{SxxR}$ involved in FMNAT activity, also common to other NTs. However, several observations can be made with respect to the $C$-terminal module in plant proteins: $i$ ) its length is 40 to 60 residues shorter; $i i)$ the PTAN motif characteristic of the RFK activity mutates to PxS; iii) a LNxPP motif is found conserved in plants, next to the invariant $\mathrm{GxY}$ motif. AtRibF1 and AtRibF2 belong to this group of proteins and were recently characterized by Sandoval et al. [30], who did not detect any RFK activity in these enzymes. This experimental observation, together with the absence of the PTAN motif suggests a different enzymatic activity for this module. Therefore we named these proteins as plant-like FADS.

Furthermore, a few bacterial parasites and pathogens isolated from plant, human or soil material and belonging to phyla Firmicutes, Actinobacteria, Tenericutes and Spirochaetes contain extra sequences with significant similarity to FADS-type I (E-values $\leq 1.5 \times 10^{-10}$ ). However, as shown in Figure 1 and 2, these sequences do not conserve the catalytic PTAN motifs, and have shorter $C$-terminal modules similar in length to plant like-FADS, suggesting that they might constitute another divergent type of FADS, which we label as FADS-type II (see Table 1).

In our sequence searches, plant-like FADS proteins are distributed across a variety of green plant lineages. Among land plants we found 84 matches (62 in Eudicots, 11 in Monocots, 6 in Coniferophyta, 3 in Magnoliids, 1 in Bryophyta, 1 in Lycopodiophyta). Other than land plants, plant-like FADS proteins were restricted to unicellular photosynthetic organisms belonging to phylum Chlorophyta (Micromonas pusilla, Chlamydomonas reinhardtii, Coccomyxa sp. and Ostreococcus lucimarinus) (see Table 2). All plant genomes surveyed encode these proteins in the nuclear genome (sequence searches in chloroplast genomes did not produce matches). Our search strategies did not find plant-like FADS proteins in any other eukaryotic genomes. These observations might imply that these genes have a prokaryotic origin, somewhat related to the endosymbiotic origin of chloroplasts. On the other hand, all green plant genomes explored have a copy of the cytosolic bifunctional FHy/ RFK protein except two Micromonas species that have a monofunctional RFK enzyme like in most eukaryotes. Proteins related to the HAD domain of FHy/RFK have been found in either bacteria or eukaryotes but this enzyme has been suggested to be unique in plant lineages probably being originated by fusion of a HAD to an eukaryotic-type RFK [29].

With the aim of further exploring the origin of plantlike FADS proteins, we carried out a phylogenetic analysis, which is summarized in the phylogram in Figure 3. According to this tree, which was rooted by taking the sequence of the cytosolic protein AtFHy/RFK from Arabidopsis thaliana as an outgroup, plant-like FADS proteins are closer to the group of cyanobacteria than any other bacterial species, which were selected to represent taxa included in Table 1. Indeed proteins from both cyanobacteria and green plants are enclosed in a clade with an associated approximate likelihood ratio (aLRT) of 0.80 . These observations suggest that plant-like FADS proteins have a prokaryotic origin closely related with cyanobacteria, although shaping a divergent group of sequences, as illustrated in Figure 3. Note that AtFHy/ RFK clusters apart from prokaryotic FADS, confirming a different origin for this enzyme. The tree also suggests that plant-like FADS proteins diverged from bacterial FADS probably before the separation of the two major plant phyla (Streptophyta -plants and their closest green algal relatives- and Chlorophyta -the rest of green algae), since they are present in species from both. In order to further investigate this, we searched for putative plant-like FADS homologues in Mesostigma viride, proposed to be the earliest plant lineage and anterior to the divergence of the Streptophyta and Chlorophyta [32]. Unfortunately, the nuclear genome of this species is not available and the chloroplast and mitochondrial genomes yielded no sequence matches. Our findings could indicate that plant-like FADS indeed derived from cyanobacterial FADS, despite the fact that they are now encoded in the nuclear genome [33].

Moreover, these results reveal that most bacteria containing FADS-type II sequences have also typical FADStype I proteins (see Table 1) and the tree in Figure 3 shows that these two types of sequences cluster together, implying that they might actually be paralogous genes. Only the genomes of Eubacterium saphenum ATCC 49989, Mycoplasma conjunctivae, Treponema pallidum subsp. Pallidum contain exclusively FADS-type II proteins. Although the tree does not support that FADStype II proteins constitute a distinct evolutionary class, their shorter and non-conserved $C$-terminal domains still clusters them clearly as a distinct functional group, which might have lost the $C$-terminal activity typical of FADS-type I proteins.

We also note the observed variability in terms of RFK and FMNAT enzymatic activities in bacterial genomes. While most prokaryotes have a single copy of a typical FADS-type I sequence, in 4 species both enzymatic activities are separated in monofunctional proteins, 


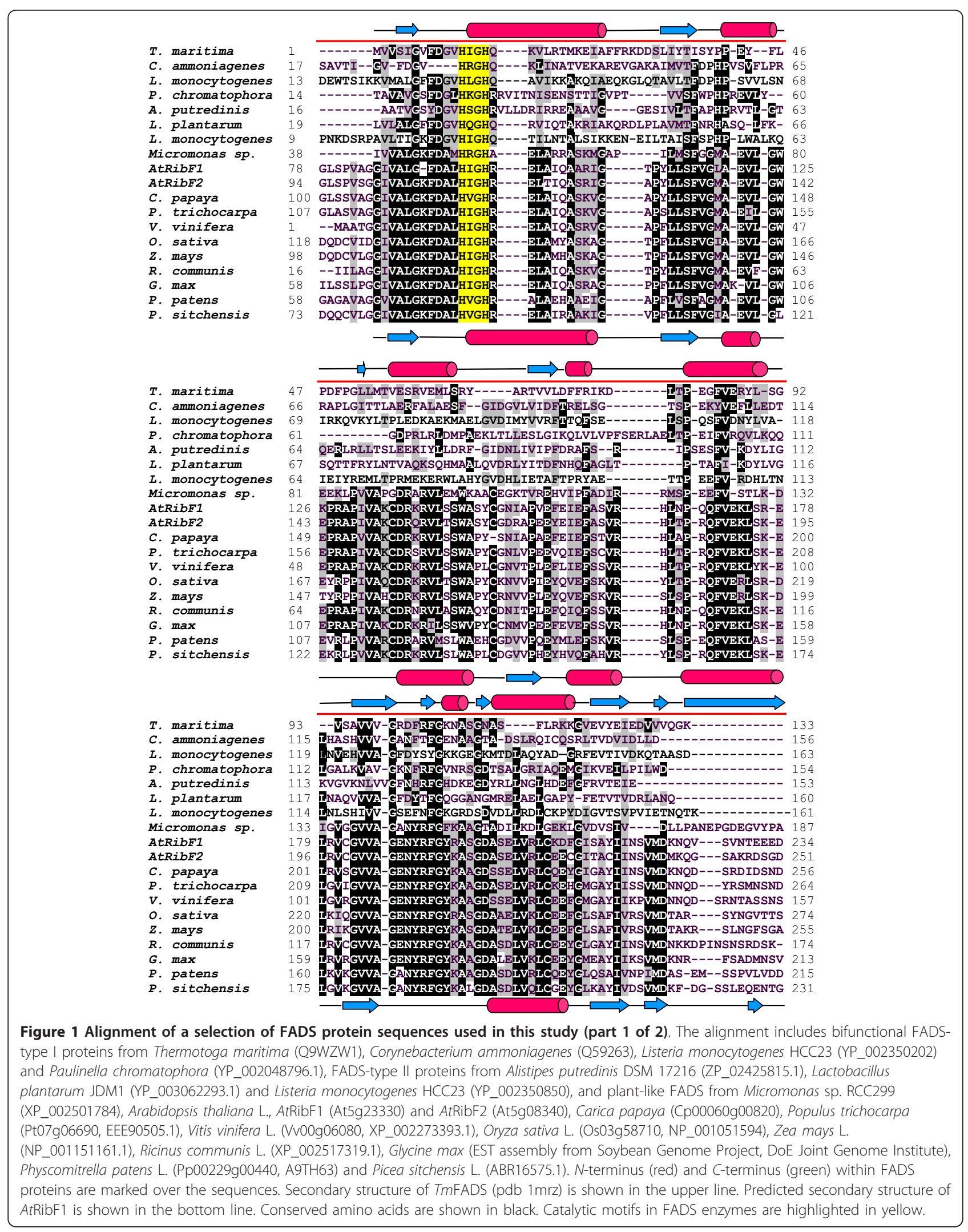




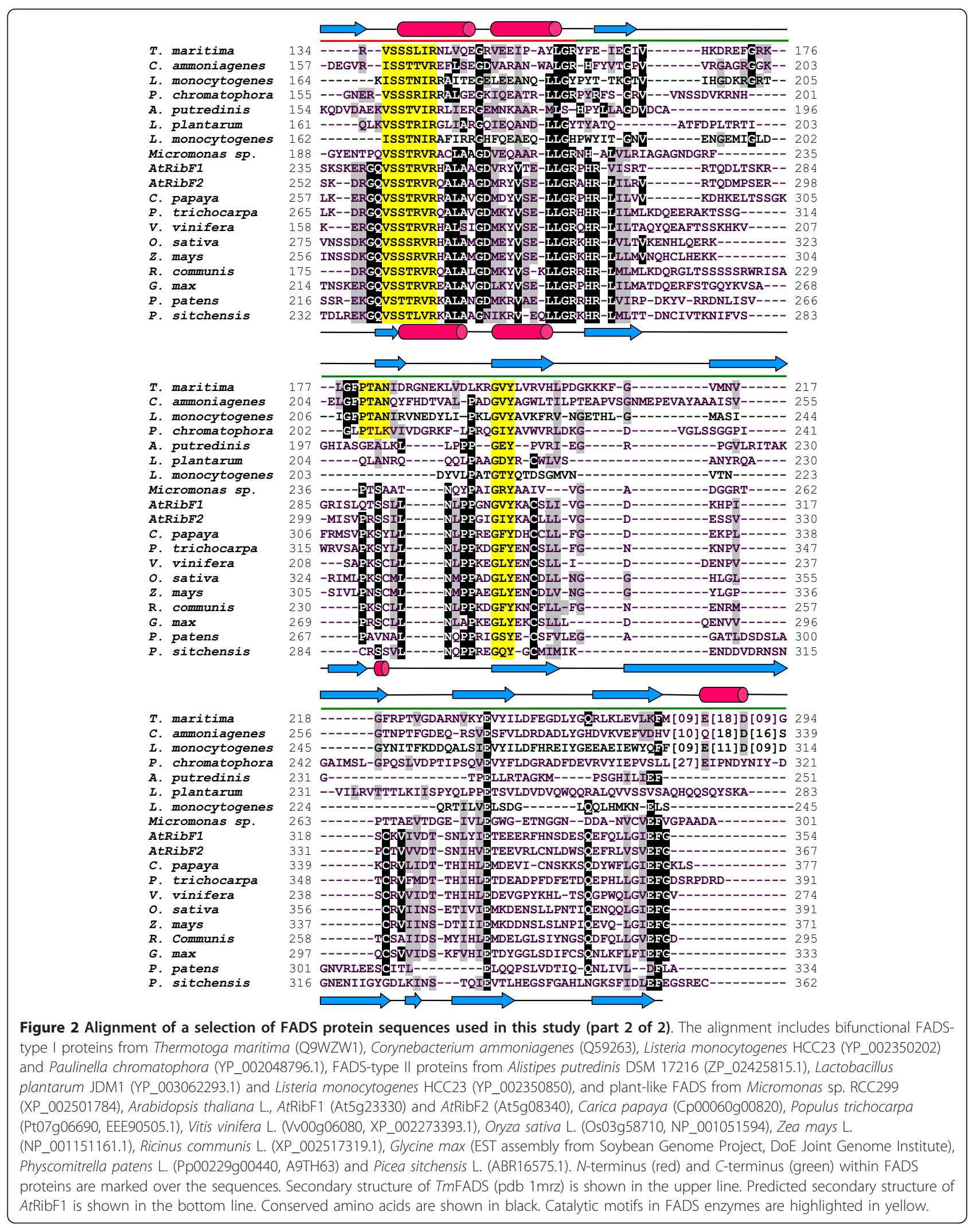


Table 2 Eukaryotic genomes containing FADS-like proteins ${ }^{(1)}$

\begin{tabular}{|c|c|c|}
\hline Type of protein & Genomes & $\mathrm{N}$ \\
\hline FADS-type I & $\begin{array}{l}\text { Anopheles gambiae } \\
\text { Babesia bovis } \\
\text { Caenorhabditis japonica strain DF5081 } \\
\text { Caernorhabditis remanei strain PB4641 } \\
\text { Culex pipiens quienquefasciatus } \\
\text { Plasmodium knowlesi } \\
\text { Trichoplax adhaerens } \\
\text { Paulinella chromatophora }\end{array}$ & 8 \\
\hline$\overline{\text { RFK }}$ & Most eukaryotic genomes & 658 \\
\hline $\begin{array}{l}\text { Plant-like FADS } \\
\text { RFK }\end{array}$ & $\begin{array}{l}\text { Micromonas pusilla } \\
\text { Micromonas sp. RCC299 }\end{array}$ & 2 \\
\hline $\begin{array}{l}\text { Plant-like FADS } \\
\text { FHy/RFK }^{(2)}\end{array}$ & $\begin{array}{l}\text { Land plant genomes } \\
\text { Chlamydomonas reinhardtii } \\
\text { Coccomyxa sp. } \\
\text { Ostreococcus lucimarinus }\end{array}$ & $87^{(3)}$ \\
\hline
\end{tabular}

${ }^{1}$ Check details in section "Sequence analysis" of Methods.

${ }^{2}$ FMN hydrolase fused to RFK in ref. 29.

${ }^{3}$ Includes unigenes compiled from ESTs.

which correspond to RFK or FMNAT modules, respectively. In other cases the FADS-type I sequence was accompanied by either monofunctional prokaryotic FMNAT (5 genomes) or monofunctional prokaryotic RFK (2 genomes). For instance, the genome of Alistipes putredinis contains both a monofunctional RFK and a FADS-type II sequence. Furthermore, although most bacterial FADS and RFK proteins include the conserved PTAN motif, some sequence variants can be found, including PTLK, PTLN, PTIN or KTAN, which nevertheless conserve the $C$-terminal module length. As these genomes do not contain any other RFK related proteins, these sequence variants are supposed still to be responsible for the RFK activity.

FADS-type I sequences were also found in 8 eukaryotic species (Table 2), including Anopheles gambiae, Caenorhabditis sp., Trichoplax adhaerens, which is considered to be the most primitive multi-cellular animal known, or the freshwater amoeba Paulinella chromatophora, which harbours a cyanobacterial endosymbiont.

It has been proposed that the double enzymatic activity of FADS proteins might be the result of a gene fusion event that genetically perpetuated an ancient proteinprotein interaction $[8,34]$. If this hypothesis holds true, it is remarkable that 1190 out of 1194 bacterial genomes have a copy of this fused gene (Table 1) while monofunctional RFKs are vastly predominant (658/755) across eukaryotic genomes (Table 2). This observed unbalance suggests that this fusion event, or functional coupling, would have been evolutionary favoured only in unicellular organisms, from which chloroplasts are thought to be derived.

We would like to remark that FADS proteins are annotated in sequence databases with confusing or contradictory names such as riboflavin biosynthesis protein RibF (i.e., YP_002487514.1), FMN adenylylate transferase (i.e., NP_692523.1), FMN adenylyltransferase (i.e., YP_001623829.1), FAD synthase (i.e., YP_518746), riboflavin kinase/FMN adenylyltransferase (i.e., YP_932710), flavokinase/FAD synthetase (i.e., YP_002783884), riboflavin kinase/FAD synthetase (i.e., NP_975116.1). Indeed, non-strictly FADS sequences are also named as that (i.e., YP_003062293.1). In the case of plants, plant-like FADS sequences are found as riboflavin kinase (i.e., gb| CO899788.1|, gb|BG509026.1|, gb|CN491424.1|) or protein-s isoprenylcysteine o-methyltransferase (i.e., PTHR12714, gb|GR935784.1)|, cassava1385). This misleading variability in names is of no benefit to users, and clearly so a consensus in the nomenclature would be desirable. We hope this work makes a contribution in this direction.

\section{A putative molecular function for the C-terminal module in plant-like FADS proteins}

PSI-BLAST searches of both the complete sequence of the plant-like FADS AtRibF1 and its $C$-terminus matched only NTs and RFKs (10 iterations, E-value $<3 \times 10^{-8}$ ) from bacteria, cyanobacteria, yeast and human. No other family was identified as related to the $C$-terminus of plant-like FADS. The similarity between the newly identified $C$-terminal module and NTs was further explored in the pdb70 structural library using the fold-recognition algorithm HHPred in local and global mode. Local searches provided significant matches (E-value $\leq 1.4 \times 10^{-17}$ ) to: the RFK-module of TmFADS (pdb 1mrz, 1s4m, 1t6x, 1t6y, 1t6z, 2i1l; [9,10]), SpRFK (pdb 1n08, 1n05, 1n07, 1n06; [13]), HsRFK (pdb 1nb0, 1nb9, 1p4m, 1q9s; [14]) and Trypanosoma brucei RFK (pdb 3bnw). Apart from these hits, global searches with the $C$-terminal domain yielded significant matches (E-values $\leq 2.5 \times 10^{-6}$ ) to: nicotinamide mononucleotide (NMN) adenylyl transferase/ribosylnicotinamide kinase from Haemophilus influenzae (pdb 1lw7), ethanolaminephosphate cytidylyltransferase from $H$. sapiens (pdb 3elb), nicotinamide-nucleotide adenylyltransferase (pdb 2qjt) from Francisella tularensis and the $C$-terminal module of bifunctional nicotinamide mononucleotide (NMN) adenylyltransferase/Nudix hydrolase from Synechocystis sp. (SyNadM-Nudix) (pdb 2qjo; [35]).

To evaluate the possible role of the $C$-terminal module of plant-like FADSs and the molecular arrangement of this protein region we modelled the $A t R i b F 1$ structure using all the above mentioned protein structures as templates. The best predicted models, as expected in terms of sequence similarity and alignment quality in putative binding and catalytic regions were obtained with the X-ray structure of TmFADS, SpRFK and HsRFK. In order to annotate putative functional residues, the comparative 


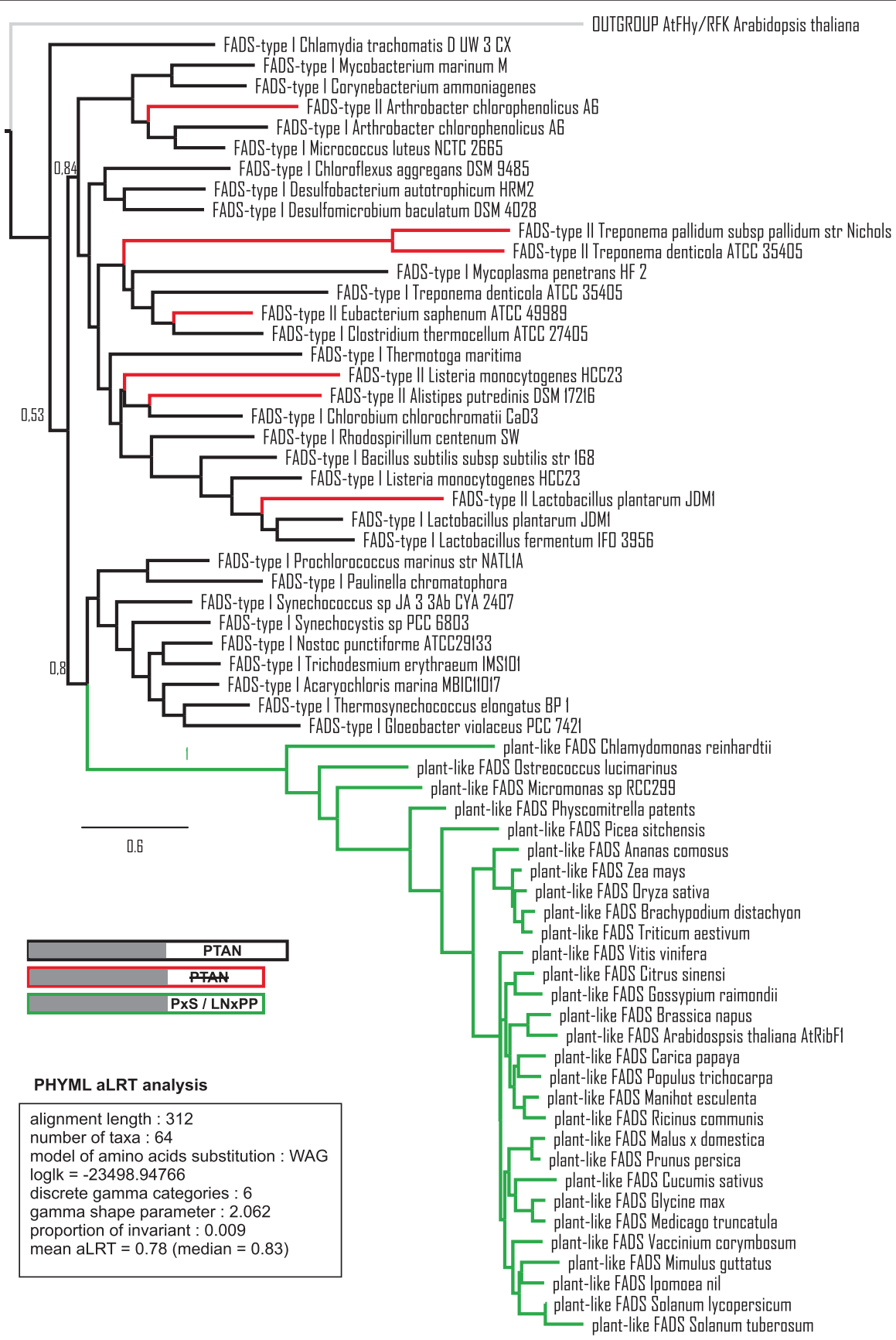

Figure 3 Maximum likelihood tree of a selection of 64 FADS sequences. Maximum likelihood tree of a selection of 64 sequences, including FADS-type I (black), FADS-type II (red) and plant-like FADS (green) proteins. Approximate likelihood ratio support values are printed next to branches and a summary of the tree parameters is shown in a box. Schematic protein representations including both the $\mathrm{N}$-terminal (in grey) and the C-terminal modules, highlighting the distinctive sequence motifs, are drawn to assist in the interpretation of the tree. The underlying multiple alignment is available in Additional file 1; Figure S3.

models were superposed to the original templates, including all ligands present in the experimental coordinates. The superposition in Figure 4 indicate that plant conserved residues ${ }^{290} \mathrm{P} \times \mathrm{S}^{292}$ and ${ }^{302} \mathrm{GVY}^{304}$ in the model occupy equivalent positions with respect to the ligands (ADP and FMN) present in the crystallographic structure of TmFADS. Similar results were obtained with $S p$ RFK and $H s$ RFK (data not shown). The predicted secondary structure of $A t \mathrm{RibF} 1$ corresponds well with that of TmFADS (see Figure 1 and 2), although it lacks the last $\alpha$-helix at the $N$-terminal end, which is conserved also in $S p$ RFK and $H s$ RFK. This helix appears to be 


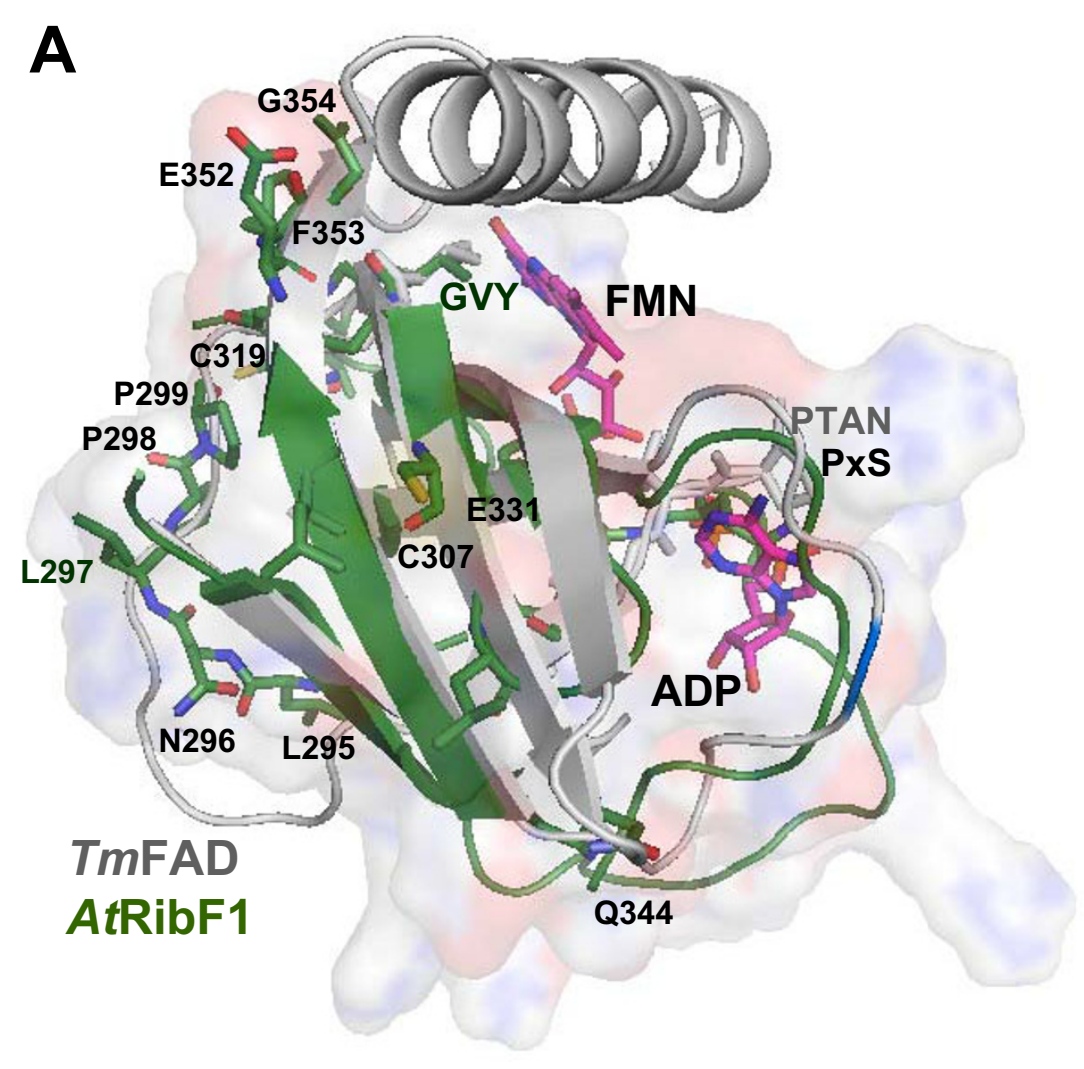

B

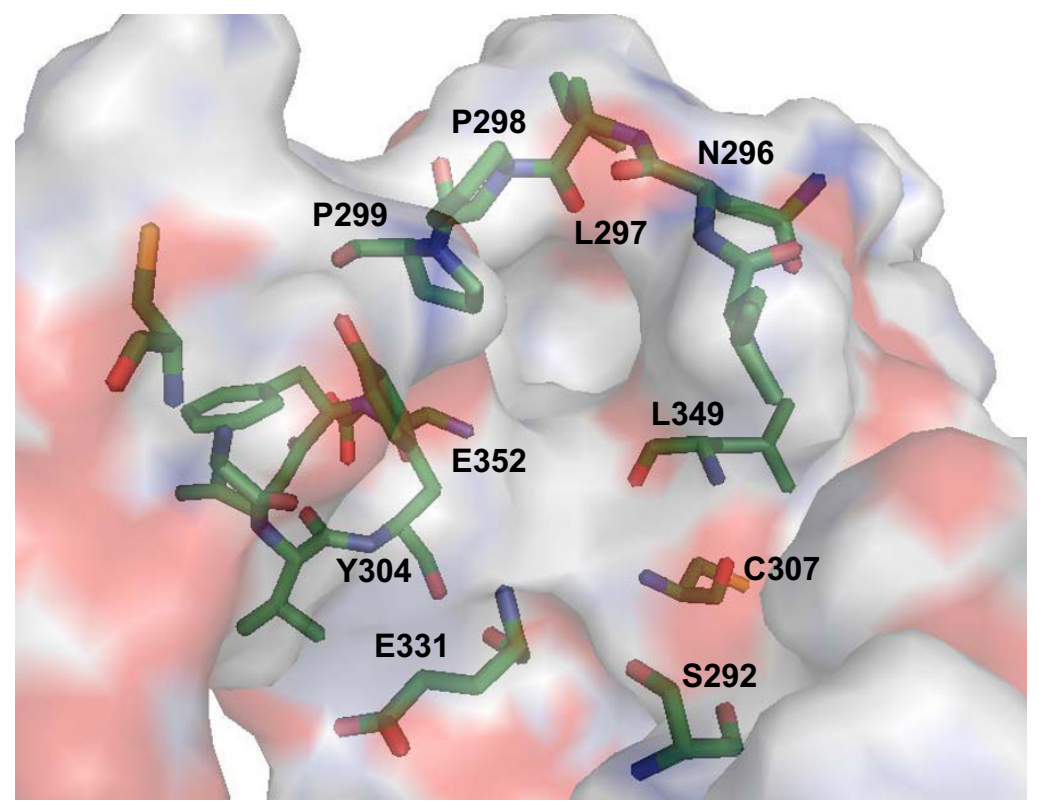

Figure 4 Structural comparison of the C-terminal module of both TmFADS (pdb 1t6y) and the homology model of AtRibF1. A) Ribbon diagrams of TmFADS (gray) and homology model of AtRibF1 (green). Bound ADP and FMN ligands in pdb 1t6y are shown as sticks in CPK with carbons in magenta. Conserved residues in the C-terminus of plant-like FADS are superposed to catalytic residues in TmFADS. Electrostatic potential surface of AtRibF1 is also shown. B) Putative new active site in the structural model of AtRibF1. Specific-plant conserved residues are shown as sticks with carbons in green. 
crucial for a correct orientation of the bound flavin substrate [14] and its absence in AtRibF1 is in agreement with the observed lack of RFK activity [30].

Figure 4 shows the specific-plant conserved residues Leu295, Asn296, Leu297, Pro298 and Pro299 $\left({ }^{295}\right.$ LNLPP $^{299}$ motif), Cys307, Cys319, Glu331, Gln344, Glu352, Phe353 and Gly354. It can be observed that the LNLPP motif is located in a flexible loop, in an opposite site of that bound FMN or ADP in TmFADS and orientated towards a cavity. Furthermore, the conserved residues Ser292, Cys307, Glu331 and Glu352 appear orientated towards this cavity suggesting that this site could possibly be a putative new binding-site in plantlike FADSs. It is also worth mentioning that Glu331 residue, invariant also in FADS and RFK families (i.e., Glu268 in FADS-type I from Corynebacterium ammoniagenes [11]), has been proposed to act as a catalytic base.

As mentioned above, remote similarity of the $C$ terminal module of plant-like FADSs was found with the $C$-terminal domain of other families such as SyNadM-Nudix $\left(\right.$ E-value $\left.=6.8 \times 10^{-08}\right)$, which belongs to a large superfamily of pyrophosphohydrolases (see Additional file 1; Figure S4). In Arabidopsis 27 Nudix hydrolase genes have been found and the proteins they encode are able to hydrolyze various types of nucleoside diphosphates derivatives such as ADP-glucose, ADPribose and a wide range of its derivatives, FAD, NADH, $\mathrm{NADPH}$, and diadenosine polyphosphates [36]. Moreover, a remote sequence consensus of this protein region including the LNxPP motif was found with serine/threonine phosphatases $2 \mathrm{C}$ and members of the hydrolase superfamily. These observations suggest that the $C$-terminal module of AtRibF1 could have a function other than RFK enzymatic activity. Sandoval et al. [30] showed that purified recombinant AtRibF1 and AtRibF2 enzymes only display FADS activity, with undetectable RFK activity and hence assumed that these are indeed monofunctional enzymes. However, they were able to measure FMN hydrolase, FAD pyrophosphatase and RFK activities in Percoll-isolated chloroplasts.

As mentioned above, our bioinformatic analyses point out that plant-like FADS proteins could be bifunctional enzymes. More precisely, structural similarities predict a hydrolase and phosphatase activity for the $C$-terminal module, although the possibility to have a nonenzymatic regulatory role or to be a simple evolutionary relic should not be dismissed. Nevertheless, considering the results of Sandoval et al. [30] and ours, we could speculate that some of the measured activities in isolated chloroplasts (i.e., FMN hydrolase or FAD pyrophosphatase) could be associated to this $C$-terminal module. In order to test this hypothesis we have designed some experiments with recombinant plant-like FADS from soybean (Glycine $\max$ ) and preliminary results seem to indicate that its $C$-terminal module might have a hydrolytic activity since GmFADS was able to convert FMN into RF (data not shown). Interestingly, this activity was not detected in purified FADS from $C$. ammoniagenes, a typical FADS-type I protein [11]. While these preliminary results seem to be in agreement with our theoretical analyses, clearly further investigations are necessary to confirm the possible enzymatic role of the $C$-terminal module of plant-like FADS. Future work will be done by using recombinant plant-like FADS (GmFADS) in order to confirm this observed hydrolytic enzymatic activity.

\section{Conclusions}

Plant-like FADS enzymes are distributed across a variety of green plant lineages and constitute a divergent protein family clearly of cyanobacterial origin. Homology models predict that plant-specific conserved residues are orientated towards a cavity, building a distinct active site when compared to that involved in substrate binding and catalysis in the $C$-terminus of typical FADS-type I enzymes. The remote relationship reported here between plant-like FADS proteins and members of pyrophosphohydrolase or phosphatase superfamilies as well as preliminary experimental results suggest that the $C$ terminal module of these proteins, clearly of bacterial origin, might be involved in a catalytic function.

\section{Methods}

\section{Sequence analysis}

The NCBI non-redundant protein sequences (nr), nucleotide collection (nr/nt) and concise microbial protein databases, and the CyanoBase (http://genome. kazusa.or.jp/cyanobase/) sequence library, were scanned with PSI-BLAST [37] and TBLASTN, in order to retrieve sequences similar (E-values $<10^{-14}$ ) to: $i$ ) FADS from Thermotoga maritima (UniProtKB Q9WZW1 $[9,10]$ ), ii) RFK from Bacillus subtilis (GenBank AAC00333.1) and iii) the plant-like FADS AtRibF1 (At5g23330, NP_568429, GenBank ACH56223.1) or AtRibF2 (At5g08340; NP_568192, GenBank ACH56224.1). To increase sensitivity, further similar sequences were retrieved by scanning either the $\mathrm{N}$ terminal or the $C$-terminal modules of prokaryotic and plant-like FADS and RFK proteins.

In order to increase the recovery of plant sequences, which are currently distributed from a variety of repositories, the AtRibF1 sequence was also scanned against JGI Genome portal (http://genome.jgi-psf.org/), Phytozome (http://www.phytozome.net/) and PLAZA (http:// bioinformatics.psb.ugent.be/plaza/) with E-values $<10^{-50}$. Finally, further searches were performed against NCBI Expressed Sequence Tags (filter: Viridiplantae) and TIGR Plant Transcript Assembly databases, with E-values $<10^{-20}$. 


\section{Multiple alignments}

The multiple alignment in Figure 1 and 2 was constructed in three steps, using the AtRibF1 protein as seed:

1) A sequence profile of plant-like proteins was compiled with ClustalW [38].

2) A selection of bacterial and eukaryotic sequences was aligned to the profile.

3) The sequence of Thermotoga maritima was added following the fold recognition alignment produced by HHPred [39] using the Protein data Bank structure $1 \mathrm{mrz}$. This template was predicted to be the best modelling template by the BioInfoBank Meta Server (see below).

The multiple alignment used to drive the phylogenetic analysis summarized in Figure 3 was constructed as follows:

1) A representative set of FADS-type I and FADS-type II sequences were multiply aligned with CLUSTALW [38] and their secondary structure was predicted with PSIPRED [40] taking the Thermotoga maritima sequence as a representative. The sequences selected are representative of bacterial species having FADStype I and/or FADS-type II, and belonging to phyla Actinobacteria, Firmicutes, Spirochaetes and Tenericutes. Also sequences from species containing only FADS-type I, which belong to phyla Chlamydiae, Chlorobi, Chloroflexi (green non-sulfur bacteria), Cyanobacteria, Proteobacteria (purple bacteria) and Thermotogae are included, providing a good coverage of diverse phylogenetic bacterial groups.

2) The sequence of the cytosolic protein AtFHy/RFK from Arabidopsis thaliana [29] was added and aligned as an outgroup, and the resulting multiple alignment was converted to a hidden Markov model in HHSearch format with hhmake [39].

3) All plant-like FADS protein sequences that covered most of both domains (from the HxGH to the GxY motif) were considered complete, aligned with CLUSTALW [38] and converted to a hidden Markov model, including the PSIPRED secondary structure prediction of $A t$ RibF1. The plant sequences selected cover the diverse phylogenetic groups of green plants as shown in Additional file 1; Figure S1.

4) The profiles 2) and 3) were globally aligned with hhalign [39] and the resulting alignment was trimmed by removing the poorly aligned segments, following the protocol "automated1" of the trimAL software (http://trimal.cgenomics.org/) [41]. The original and trimmed alignments are available in Additional file 1; Figures S2 and S3.

\section{Phylogenetic analysis}

The trimmed multiple alignment described above was used to drive a maximum likelihood phylogenetic tree with PhyML [42] and the best fitting amino acid substitution model selected with ProtTest [43]. The tree was midpoint-rooted and plotted with FigTree (http://tree. bio.ed.ac.uk/software/figtree).

\section{Molecular modelling}

To further investigate possible molecular functions of the $C$-terminal module of plant-like FADS proteins the complete protein sequence of $A t$ RibF1 as well as its $C$-terminal domain were submitted to the BioInfoBank Meta Server [44]. The best aligned template provided by FUGUE [45] and PSI-BLAST [37] were subsequently employed to drive homology modelling with Modeller [46]. Further templates were found with HHpred [39] scans of the pdb70 library. Structural superposition and alignments were performed with the software MAMMOTH [47]. Molecular structures and models were inspected, analyzed and plotted with PyMol [48]. Secondary structure predictions were made with PSIPRED [40].

\section{Production of GmFADS and activity measurement}

GmFADS gene synthesis, and E. coli protein overexpression and purification were carried out by GeneScript USA Inc. Conversion of FMN into RF was qualitatively assayed by addition of GmFADS or CaFADS [11] (final enzyme concentration $\sim 0.2 \mu \mathrm{M}$ ) to a solution (final volume, $150 \mu \mathrm{l}$ ) containing $50 \mu \mathrm{M}$ FMN, either 0 or $0.2 \mathrm{mM}$ ATP and $10 \mathrm{mM} \mathrm{MgCl}_{2}$, in $50 \mathrm{mM}$ Tris$\mathrm{HCl}, \mathrm{pH}$ 8.0. After incubation overnight at $25^{\circ} \mathrm{C}$ or 5 $\mathrm{min}$ at $37^{\circ} \mathrm{C}$ the reaction was stopped by boiling the preparations for 5 minutes. Transformation of FMN was visualized by resolving the products of the reaction at room temperature and in the dark by TLC on Silica Gel SIL-G-25 $(20 \mathrm{~cm} \times 20 \mathrm{~cm}$, thickness $0.25 \mathrm{~mm})$ plates. The moving phase was a solution of butanol:acetic acid: water (12:3:5). Flavin TLC spots were visually examined and scanned by determining their fluorescence under an ultraviolet light [11].

\section{Additional material}

Additional file 1: Additional Figures. Figure S1.- Taxonomy of plantlike FADS sequences used in the phylogenetic analysis presented in the main text (Figure 3). Figure S2.- Multiple alignment of a representative set of FADS-type I, FADS-type II and plant-like FADS protein sequences. The alignment was obtained, as explained in Methods, to drive the phylogenetic analysis presented in the paper. Figure S3.- Trimmed multiple alignment of the set of FADS-type I, FADS-type II and plant-like FADS protein sequences used to build the PHYML maximum likelihood tree in Figure 3 of the paper. The alignment was trimmed with the 'automated1' option of the trimAl software. Figure S4.- HHPred alignment of structural template SyNadMNudix (pdb 2qjo) and AtRibF1. 


\section{List of Abbreviations}

FADS-type I: bifunctional prokaryotic enzyme with riboflavin kinase and FMN adenylyltransferase activities; FADS-type II: prokaryotic enzyme with FMN adenylyltransferase activity of FADS in the $\mathrm{N}$-terminal module and a putative different activity in the C-terminal module; FMNAT: monofunctional prokaryotic enzyme with FMN adenylyltransferase activity; FMNAT-module: module of FADS with FMN adenylyltransferase activity; plant-like FADS: bifunctional enzyme found in plants with FMN adenylyltransferase activity of FADS in the $\mathrm{N}$-terminal domain and a putative different activity to that of FADS-type I in the C-terminal domain; RFK: monofunctional prokaryotic enzyme with riboflavin kinase activity; RFK-module: module of FADS with riboflavin kinase activity; Tris: Tris (hydroxymethyl)aminomethane.

\section{Acknowledgements}

We thank L. Sánchez-Pulido, P. Vinuesa and D. Moreno for comments on the manuscript. S. Arilla-Luna holds a fellowship from the Spanish Ministry of Science and Innovation (FPU program). This work was supported by CONSI +D, DGA (Grant PM062/2007 to M.M. and I.Y.), the Spanish Ministry of Science and Innovation (BIO2007-65890-C02-01 and BIO2010-14983 to M.M.) and Gobierno de Aragón (DGA-GE B18 to M. M. and I.Y. and DGA-GC A06 to B.C-M).

\section{Author details}

'Estación Experimental de Aula Dei, Consejo Superior de Investigaciones Científicas (CSIC), Avda. Montañana, 1005, 50059 Zaragoza, Spain. ${ }^{2}$ Institute of Biocomputation and Physics of Complex Systems (BIFI), Universidad de Zaragoza, Mariano Esquillor, Edificio I + D, 50018 Zaragoza, Spain. ${ }^{3}$ Departamento de Bioquímica y Biología Molecular y Celular, Facultad de Ciencias, C/ Pedro Cerbuna 12, 5009 Zaragoza, Spain. ${ }^{4}$ Fundación ARAID, Zaragoza, Spain.

\section{Authors' contributions}

IY carried out the sequence analysis, sequence alignment and molecular modelling, participated in the design and coordination of the study, and drafted the manuscript. BCM participated in the design of the study, performed the phylogenetic analysis, and helped write the manuscript. SAL participated in the preliminary experimental studies. MM conceived the study, and participated in its design and helped draft the manuscript. All authors have read and approved the final manuscript.

Received: 30 April 2010 Accepted: 18 October 2010

Published: 18 October 2010

\section{References}

1. Müller F: Chemistry and Biochemistry of Flavoenzymes.Edited by: Müller F. CRC PressBoca Raton, FL; 1991:1:1-77.

2. Powers HJ: Riboflavin (vitamin B-2) and health. Am J Clin Nutr 2003, 77:1352-1360.

3. Joosten V, van Berkel WJ: Flavoenzymes. Curr Opin Chem Biol 2007, 11:195-202.

4. Van Berkel WJH: Chemistry of flavoenzymes. Wiley Encyclopedia of Chemical Biology John Wiley \& Sons, Inc, Hoboken, NJ 2008.

5. Merrill AH Jr, Lambeth JD, Edmondson DE, McCormick DB: Formation and mode of action of flavoproteins. Annu Rev Nutr 1981, 1:281-317.

6. Bacher A, Eberhardt S, Fischer M, Kis K, Richter G: Biosynthesis of vitamin B2 (Riboflavin). Annu Rev Nutr 2000, 20:153-167.

7. Roje S: Vitamin B biosynthesis in plants. Phytochemistry 2007, 68:1904-1921.

8. Krupa A, Sandhya K, Srinivasan N, Jonnalagadda S: A conserved domain in prokaryotic bifunctional FAD synthetases can potentially catalyze nucleotide transfer. Trends Biochem Sci 2003, 28:9-12.

9. Wang W, Kim R, Jancarik J, Yokota H, Kim S-H: Crystal structure of a flavinbinding protein from Thermotoga maritima. Protein Struct Funct Genet 2003, 52:633-635.

10. Wang W, Kim R, Yokota H, Kim S-H: Crystal structure of a flavin-binding to FAD synthetase of Thermotoga maritima. Protein Struct Funct Genet 2005, 58:246-248.

11. Frago S, Martínez-Júlvez M, Serrano A, Medina M: Structural analysis of FAD synthetase from Corynebacterium ammoniagenes. BMC Microbiology 2008, 8:160-175.
12. Herguedas B, Martínez-Júlvez $M$, Frago $S$, Medina M, Hermoso JA: Crystallization and preliminary X-ray diffraction studies of FAD synthetase from Corynebacterium ammoniagenes. Acta Cryst Section F Structural Biology and Crystallization Communications 2009, 65:1285-1288

13. Bauer S, Kemter K, Bacher A, Huber R, Fischer M, Steinbacher S: Crystal structure of Schizosaccharomyces pombe riboflavin kinase reveals a novel ATP and riboflavin-binding fold. J Mol Biol 2003, 326:1463-1473.

14. Karthikeyan S, Zhou Q, Mseeh F, Grishin NV, Osterman AL, Zhang H: Crystal structure of human riboflavin kinase reveals a $\beta$ barrel fold and a novel active site arch. Structure 2003, 11:265-273.

15. Solovieva IM, Kreneva RA, Leak DJ, Perumov DA: The ribR gene encodes a monofunctional riboflavin kinase which is involved in regulation of the Bacillus subtilis riboflavin operon. Microbiology-SGM 1999, 145:67-73.

16. Clarebout G, Villers C, Leclerca R: Macrolide resistance gene mreA of Streptococcus agalactiae encodes a flavokinase. Antimicrob Agents Chemother 2001, 45:2280-2286.

17. Kashchenko VE, Shavlovskii GM: Purification and properties of riboflavin kinase of pichia-guilliermondii. Biochemistry-Moscow 1976, 41:313-319.

18. Santos MA, Jiménez A, Revuelta JL: Molecular characterization of FMN1, the structural gene for the monofunctional flavokinase of Saccharomyces cerevisiae. J Biol Chem 2000, 275:28618-28624

19. Wu M, Repetto B, Glerum DM, Tzagoloff A: Cloning and characterization of FAD1, the structural gene for flavin adenine dinucleotide synthetase of Saccharomyces cerevisiae. Mol Cell Biol 1995, 15:264-271.

20. McCormick DB, Oka M, Bowers-Komro DM: Purification and properties of FAD synthetase from liver. Methods Enzymol 1997, 280:407-413.

21. Huerta C, Borek D, Machius M, Grishin NV, Zhang H: Structure and mechanism of an eukaryotic FMN adenylyltransferase. J Mol Biol 2009, 389:388-400.

22. Brizio C, Gallucio M, Wait R, Torchetti EM, Bafunno V, Accardi R, Gianazza E, Indiveri C, Barile M: Over-expression in Escherichia coli and characterization of two recombinant isoforms of human FAD synthetase. Biochem Biophys Res Comm 2006, 344:1008-1016

23. Torchetti EM, Brizio C, Colella M, Galluccio M, Giancaspero TA, Indiveri C, Roberti M, Barile M: Mitochondrial localization of human FAD synthetase isoform 1. Mitochondrion 2010, 10:263-273.

24. Sobhanaditya J, Rao NA: Affinity-chromatographic procedure for the purification of the enzyme from mung-bean (Phaseolus aureus) seeds and conformational-changes on its interaction with ortho-phosphate. Biochem J 1981, 197:227-232

25. Sadasivam S, Shanmugasundaram ER: Studies on flavokinase of Solanum nigrum L. Enzymologia 1966, 31:203-208

26. Giri KV, Krishnaswamy PR, Rao NA: Occurrence of flavokinase activity in plants. Nature 1957, 179:1134-1135.

27. Giri KV, Rao NA, Cama HR, Kumar AS: Studies on flavinadenine dinucleotide-synthesizing enzyme in plants. Biochem J 1960, 75:381-386

28. Mitsuda $\mathrm{H}$, Tsuge $\mathrm{H}$, Tomozawa $\mathrm{Y}$, Kawai F: Multiplicity of acid phosphatase catalyzing FMN hydrolysis in spinach leaves. J Vitaminol (Kyoto) 1970, 16:52-57.

29. Sandoval FJ, Roje S: An FMN hydrolase is fused to riboflavin kinase homolog in plants. J Biol Chem 2005, 280:38337-38345.

30. Sandoval FJ, Zhang Y, Roje S: Flavin nucleotide metabolism in plants: Monofunctional enzymes synthesize FAD in plastids. J Biol Chem 2008 283:30890-30900.

31. Giancaspero TA, Locato V, de Pinto MC, de Gara L, Barile M: The occurrence of riboflavin kinase and FAD synthetase ensures FAD synthesis in tobacco mitochondria and maintenance of cellular redox status. FEBS J 2009, 276:219-231.

32. Lemieux C, Otis C, Turmel M: Ancestral chloroplast genome in Mesostigma viride reveals an early branch of green plant evolution. Nature 2000, 403:649-652.

33. Martin W, Rujan T, Richly E, Hansen A, Cornelsen S, Lins T, Leister D, Stoebe B, Hasegawa M, Penny D: Evolutionary analysis of Arabidopsis, cyanobacterial and chloroplast genomes reveals plastid phylogeny and thousands of cyanobacterial genes in the nucleus. Proc Natl Acad Sci USA 2002, 99:12246-12251.

34. Marcotte EM, Pellegrini M, Ng HL, Rice DW, Yeates TO, Eisenberg D: Detecting protein function and protein-protein interactions from genome sequences. Science 1999, 285:751-753. 
35. Huang N, Sorci L, Zhang X, Brautigan C, Li X, Raffaelli N, Magni G, Grishin NV, Osterman A, Zhang H: Bifunctional NMN adenylyltransferase/ ADP ribose pyrophosphatase: structure and function in bacterial NAD metabolism. Structure 2008, 16:196-209.

36. Ogawa T, Yoshimura K, Miyake H, Ishikawa K, Ito D, Tanabe N, Shigeoka S: Molecular characterization of organelle-type Nudix hydrolases in Arabidopsis. Plant Physiol 2008, 148:1412-1424.

37. Altschul SF, Madden TL, Schaffer AA, Zhang J, Zhang Z, Miller W, Lipman DJ: Gapped BLAST and PSI-BLAST: a new generation of protein database search programs. Nucl Acids Res 1997, 25:3389-3402.

38. Thompson JD, Higgins DG, Gibson TJ: CLUSTAL W: improving the sensitivity of progressive multiple sequence alignment through sequence weighting, position-specific gap penalties and weight matrix choice. Nucl Acids Res 1994, 22:4673-4680.

39. Söding J, Biegert A, Lupas AN: The HHpred interactive server for protein homology detection and structure prediction. Nucl Acids Res 2005, 33: W244-W248.

40. McGuffin LJ, Bryson K, Jones DT: The PSIPred protein structure prediction server. Bioinformatics 2000, 16:404-405.

41. Capella-Gutierrez S, Silla-Martínez JM, Gabaldón T: trimAl: a tool for automated alignment trimming in large-scale phylogenetic analyses. Bioinformatics 2009, 25:1972-1973.

42. Guindon S, Gascuel O: A simple, fast and accurate algorithm to estimate large phylogenies by maximum likelihood. Syst Biol 2003, 52:696-704.

43. Abascal F, Zardoya R, Posada D: ProtTest: Selection of best-fit models of protein evolution. Bioinformatics 2005, 21:2104-2105

44. Ginalski K, Elofsson A, Fischer D, Rychlewski L: 3D-Jury: a simple approach to improve protein structure predictions. Bioinformatics 2003, 19:1015-1018.

45. Shi J, Blundell TL, Mizuguchi K: FUGUE: Sequence-structure homology recognition using environment-specific substitution tables and structure-dependent gap penalties. J Mol Biol 2001, 310:243-257.

46. Sali A, Blundell AT: Comparative protein modelling by satisfaction of spatial restraints. J Mol Biol 1993, 234:779-815.

47. Ortíz AR, Strauss CE, Olmea O: MAMMOTH (matching molecular models obtained from theory): an automated method for model comparison. Protein Sci 2002, 11:2606-2621.

48. DeLano WL: The PyMOL User's Manual. DeLano Scientific, Palo Alto, CA, USA 2002.

doi:10.1186/1471-2148-10-311

Cite this article as: Yruela et al.: Evolutionary divergence of chloroplast FAD synthetase proteins. BMC Evolutionary Biology 2010 10:311.

\section{Submit your next manuscript to BioMed Central and take full advantage of:}

- Convenient online submission

- Thorough peer review

- No space constraints or color figure charges

- Immediate publication on acceptance

- Inclusion in PubMed, CAS, Scopus and Google Scholar

- Research which is freely available for redistribution

Submit your manuscript at www.biomedcentral com/submit 